\section{Avitaminosis A y trastornos de desarrollo dentoalveolar}

\author{
Avitaminosis A \& dentoalveolar development disturbance
}

\section{Resumen}

Numerosas investigaciones han demostrado los efectos adversos de la hipovitaminosis A en el campo estomatológico, caracterizados por atrofia de las células ectomesenquimales y desordenada hiperactividad osteoblástica durante la fase del desarrollo embrionario; sin cmbargo, es limitada la información acerca de las manifestaciones histológicas por deficiencia en etapas posteriores al nacimiento; teniendo en cuenta que somos un país en desarrollo con problemas nutricionales no resueltos

El presente estudio se realizó con el objetivo de establecer los cambios morfológicos del complejo dentoalveolar presentes, después del nacimiento, por deficiencia de vitamina A; para lo cual, se empleó 20 ratas Holstman, de 21 días de cdad, alimentados con dieta carente de vitamina A. F.1 grupo control en adición recibió semanalmente una dosis de 100 U.I. de retinol a fin de completar el balance dietético normal Los cortes histologicos obtenidos por métodos convencionales, después de 7 y 8 semanas de fase experimental, de incisivos de rata con avitaminosis $A$, mostraron en la basc odontogénica, aposición adamantina ácido resistente, que va de 0 a $3 \mu$ de espesor (Fig.3), con ameloblástos cilindricos funcionalmente inhibidos; en algunos casos, con pérdida de sus conexiones plasmáticas laterales. Además, presenta diferenciación de odontoblastos bucales con reducida producción de matriz dentinaria y ausencia de odontoblastos linguales (Fig.3). La pulpa dentaria adyacente a las células especializadas es mesenquimatosa y vascularizada (Fig.3).

Los cortes longitudinales y transversales coronarios de molares de rata con avitaminosis $A$, muestran un complejo dentinopulpar con escasa producción de predentina, en algunos casos no existe. El complejo periodontal mostró un aparente engrosamiento de la cresta alveolar, sin mucstras de remodelación (Fig.4). Los cambios morfologicos del complejo dentoalveolar presente en la avitaminosis A, probado con chi cuadrado a un nivel de confianza del $99 \%$, es altamente significativa (ver graficos 1-7).

\section{Abstract}

Several investigations have demonstrated the adverse effects of the hypovitaminosis A in the stomatological field, characterized by ectomesenchymal cells atrophy and osteoblastic hyperactivity disordered during the phase of the embryonic development; however, the information is limited to the histological manifestations by deficiency in later stages after birth; keeping in mind that we are a country in development with nutritional problems not resolved

The present study was carried out to establish the morphological changes of the dentoalveolar complex present, after birth, for vitamin A deficiency; for which, we used 20 Holstman rats, of 21 days of age, fed with diet of lacking vitamin $A$, The control group, in addition received weekly one dosis of 100 U.I. of retinol at last to complete the normal dictetic balance.

The histological cuts, of rat incisors with avitaminosis A, colored with hematoxilyn and eosin, obtained by conventional methods after the seven and eigth week of experimental phase, Shown in the odontogenic base acid resistant enamel apposition which goes from 0 to $3 \mu$ of thickness (fig. 3), with cylindrical ameloblasts inhibited funcionally and in some cases with lost of their plasmatic union complex side conextion.

Besides, it shows differentiation of bucal adontoblasts with reduced production of dentine matrix and absence of lingual odontoblasts (fig.3), the dental pulp next to the specialized cells is mesenquimatic and vascularized (Fig.3).

The transverse and longitudinal histological cuts of the molars crown with avitaminosis $\mathrm{A}$, shown a pulpodentinary complex with lack of production of predentine, and in some cases almost unexistant. The periodontal complex shown a relative enlargement of the alveolar crest without sign of remodelation (fig.4). The morfological changes of the dentoalveolar complex existent in cases of avitaminosis $\mathrm{A}$, tested with Chi Square at a level of $99 \%$ confidence is significant high (see graphics 1-7).

\author{
Luis H. Gálvez Calla ${ }^{1 *}$; Guido Ayala \\ Maced o ${ }^{2^{*}}$ y Marieta Petkova \\ Gueorguieva ${ }^{3 *}$ \\ 1.3 Departamento Académico de Ciencias \\ Básicas, Facultad de Odontologia \\ 2 Facultad de Medicina \\ * Universidad Nacional Mayor de San Mar. \\ cos, Lima-Perú \\ E-mail: Igalvezc@unmsm.eciu.pe
}

Palabras clave: Complejo dentoalveolar, complejo dentinopulpar, dentina, avitaminosis $A$, histología dental
Key worils: Dentoalveolar complex, dentinopulpar complex, dentine, avitaminosis $\mathrm{A}$, dental histology 


\section{Introducción}

Además del papel que cumple en la visión, la vitamina $A$ interviene en $\mathrm{mu}-$ chos otros procesos fisiológicos. A la luz de los conocimientos actuales, esta claro su participación en el metabolis$\mathrm{mo}^{1,2,3}$, en la respuesta inmunológica, en la espermatogénesis, en el desarrollo fetal, en el gusto, en la audición, en el apetito, y en el crecimiento. Casi todos ellos dependen directa e indirectamente de la diferenciación celular ${ }^{5}$.

La deficiencia de vitamina A en etapas tempranas del crecimiento y desarrollo produce un completo cese de las actividades biológicas proliferativas ${ }^{6}$, además altera la función de fagocitosis $y$ de lisis de los macrófagos para responder a las agresiones microbianas ${ }^{7}$.

Numerosas investigaciones ${ }^{8-16}$ han demostrado los efectos adversos de la hipovitaminosis A en el campo estomatológico, caracterizados por atrofia de las células ectomesenquimales y desordenada hiperactividad osleoblástica durante la fase del desarrollo embrionario, originando irregularidades en la actividad osteoclástica en un intento infructuoso de controlar la actividad osteoblástica ${ }^{11,12}$. En casos graves, observaron hipoplasia o ausencia del esmalte por alteraciones en el estrato ameloblástico, desorientación de odontoblástos y formación de dentina defectuosa ${ }^{8,9}$. Fstos resultados fueron confirmados ampliamente por otras investigaciones ${ }^{10,14}$. Además reportaron defectos en la estructura de la dentina, con cámara pulpar desplazada hacia lingual, atrofia del órgano adamantino $y$ metaplasia de ameloblástos ${ }^{10}$.

De manera que, la diferenciación celular, una de las funciones más importantes de la vitamina $A$, no solo está limitada al organismo en desarrollo, sino que también participa activamente en el mantenimiento celular.

Estudios experimentales ${ }^{21}$ en perros alimentados con dietas carentes de vitamina $A$, han mostrado engrosamiento del tejido gingival, caracterizado por aumento en el grado de queratinización del epitelio y una mayor condensación fibrilar de tipo elástico en el conectivo; hecho que sugiere importante la presencia de micronutrientes en la dieta, no solo antes sino después del nacimiento, para mantener adecuadamente saludables a los tejidos epiteliales y conjuntivos. Sin embargo, es limitada la información acerca de sus efectos en los tejidos dentarios; lo que motivó estudiar histológicamente a los trastornos de desarrollo dentoalveolares por deficiencia de vitamina A en la dieta.

\section{Material y Método}

Se empleó 20 ratas albinas machos de raza Holstman seleccionados aleatoriamente por orden de destete en el dia, después de 21 dias de haber nacido, alimentados durante 7 a 8 semanas con dieta carente de vitamina A y agua ad libitum. El grupo control, recibió además una dosis semanal de 100 UI de retinol vía sonda faríngea, con el fin de completar el balance dietético normal

Al cabo del cual, las regiones mandibulares fueron disecadas $y$ tratadas con los métodos convencionales para el procesamiento histológico, cuyos cortes longitudinal y transversales del complejo dentoalveolar de molares y longitudinalmente de incisivos fueron colorearon con hematoxilina y eosina y examinados al microscopio óptico.

\section{Resultados}

Los cortes longitudinales de incisivo de ratas con avitaminosis $A$, luego de 7 a 8 semana de experimentación, muestran ameloblastos poco activos, más o menos cilindricos que en algunos casos pierden sus conexiones plasmáticas laterales distales, con aposición adamantina que va de $0 \mu$ a $3 \mu$ de espesor (Fig.1); en comparación con los casos

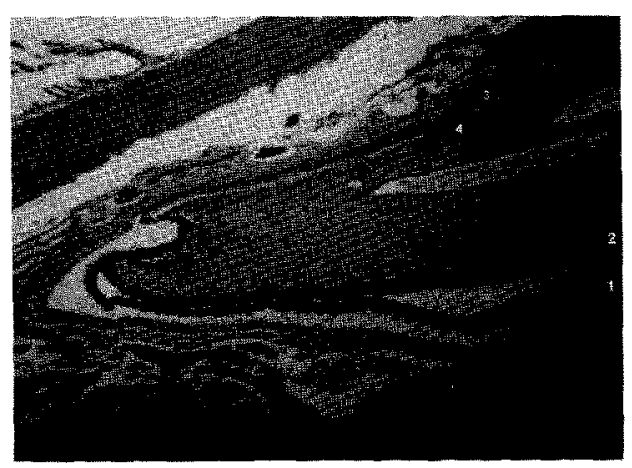

Fig. 1: Corte longitudinal de in cisivo de rata con avitaminosis A, luego de 7 a 8 semanas de experimentación, presenta: epitelio ameloblástico a cèlulas cilindricas(a) con reducida aposición adamantina (e), odontoblástos bucales diferenciados (o) con reducida aposición dentinaria (d), ausencia de odontoblastos linguales $y$ dentina. Pulpa mesenquimá tica vascularizada. Aumento 20x, H.E.

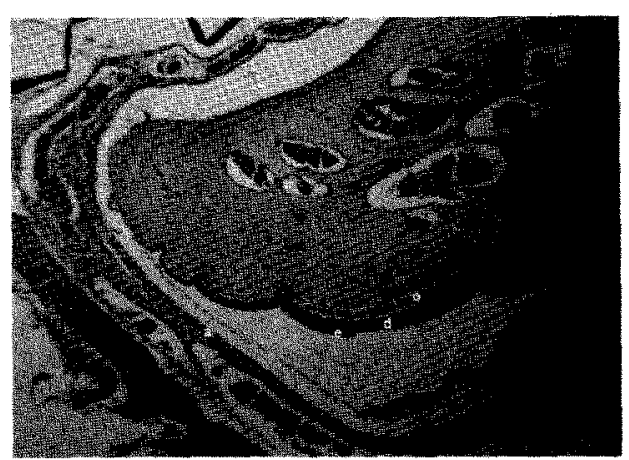

Fig.2: Corte longitudinal de incisivo de rata con dieta normal, luego de 7 a 8 semana de experimentación. Muestra zona odontogénica. Lado bucal: aposición adamantína (1) y dentinaria (2). Lado lingual: una capa dentinaria (3) más delgada junto a odontoblástos(4) más o menos rilíndricos. La pulpa es mesenquimática y vascularizada. Aumento 20 $x, H . E$. 
de incisivos (Fig.3) a diferencia del grupo control que presentan signos de activa remodelación ósea (Fig.4). El tejido conectivo de la mucosa gingival es a delgados haces de fibras colágenas. En ratas normales la mucosa gingival esta constituida por un tejido conectivo

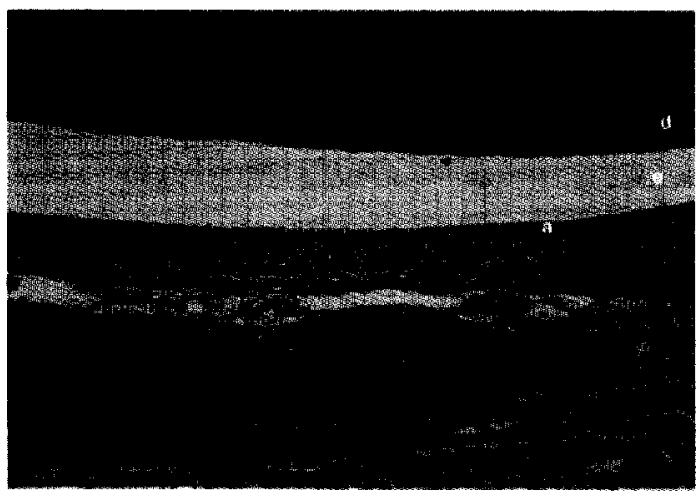

Fig.3: Corte longitudinal de incisivo de rata con avitaminosis $\mathrm{A}$, luego de 7 a 8 semanas de experimentación. Se observa: dentina (d), esmalte (e) (espacio), epitelio ameloblástico (a), hueso alveolar (h) aparentemente engrosado sin muestras de remodelación. Aumento 200x, H.E.

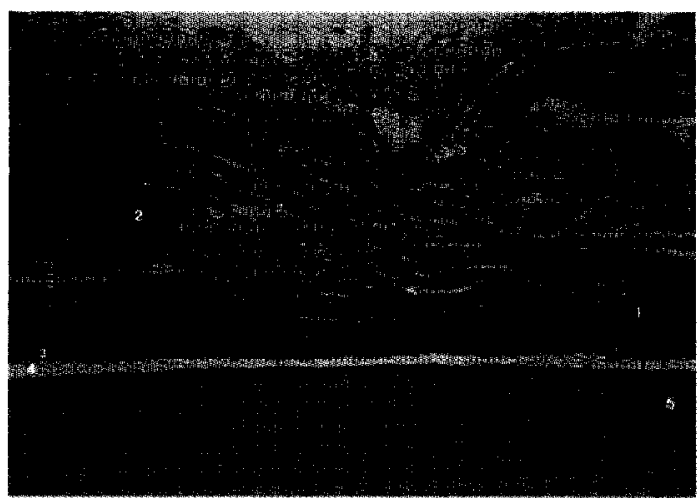

Fig.4 Corte longitudinal de incisivo de rata con dieta normal, luego de 7 a 8 semanas de experimentación: Muestra el lado bucal del complejo periodontal a nivel de cresta ósea. Se observa: conectivo denso de la mucosa gingival a gruesos haces de fibras colágenas (1).Cresta alveolar con superficie interna irregular (2). Epitelio ameloblástico con células cilindricas bajas y cúbicas (3). Esmalte (4)(espacio). Dentina (5) Aumento 200x,H.E.

Gráfico 3a. Cambios morfológicos en el odontoblasto lingual

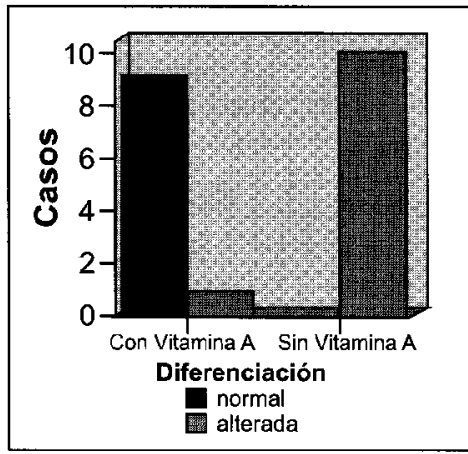

Es siginifcante la diferenciación del odontoblasto lingual en estados carenciales de Vitamina A

* $\mathrm{x}^{2} \mathrm{obs}=12.929 \mathrm{p}=0.00032$ Fisher $=0.000059$ $p<0.01$

* Con factor de correción.

Gráfico 3B. Cambios morfológicos en el odontoblasto bucal

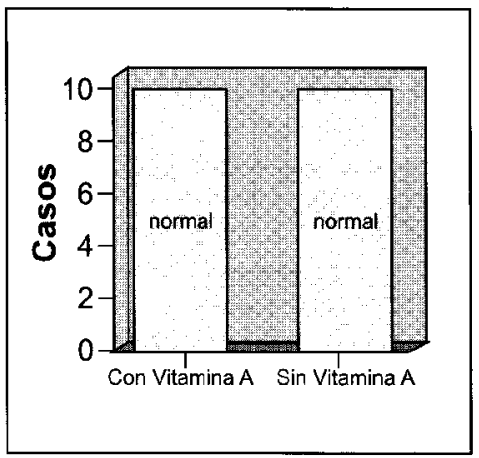

La falla deVitamina A No altera significativamente la diferenciación del odontoblasto bucal n la base odontogénica

* $x^{2}$ obs $=0 \quad p>0.01$ Existe diferenciación, pero son menos activos.

* Con factor de correción.

Gráfico 1. Cambios morfológicos del ameloblasto

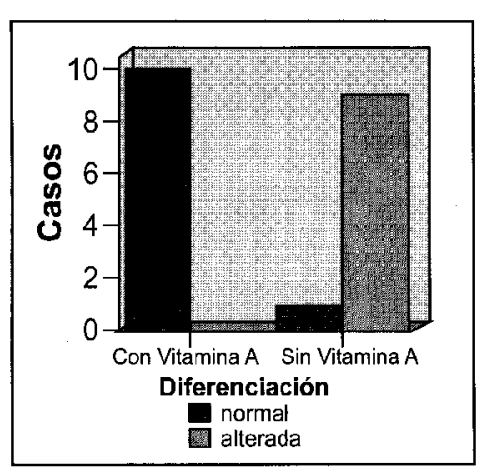

* $x^{2}$ obt $=12.929 p=0.00032$ Fisher $=0.000059$ $\mathrm{p}<0.01$

* Con factor de correción. Es significante la alteración morfolófica del ameloblasto
Gráfico 2. Cambios morfológicos en la matriz adamantina

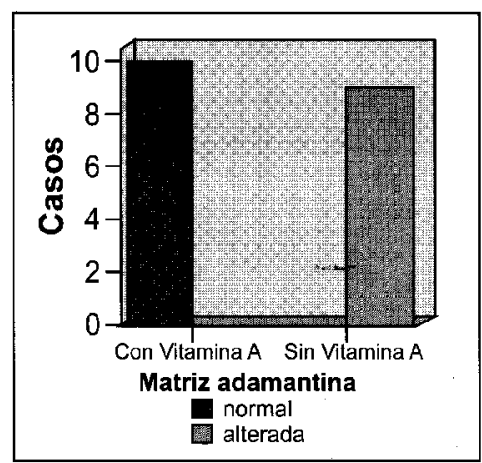

Es siginifcante la inhibición de la síntesis de matriz adamantina en la base odontogénica por carencia de Vitamina A

* $x^{2}$ obs $=16.200 p=0.0000256$ Fisher $=$ $0.0000054 \mathrm{p}<0.01$

* Con factor de correción.
Gráfico 4a. Cambios morfológicos en la matriz dentiaria lingual

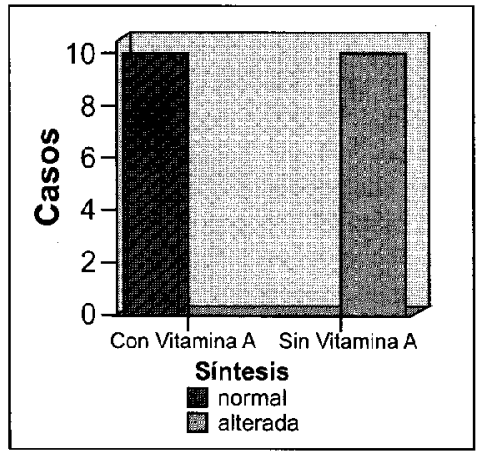

Es siginifcante la alteración de síntesis dentiaria de la base odontogénica en estados carenciales de Vitamina A

* $x^{2}$ obs $=16.200 p=0.000056$ Fisher $=$ $0.0000054 p<0.01$

* Con factor de correción. 
Gráfico 4ab. Cambios morfológicos en la matriz dentiaria

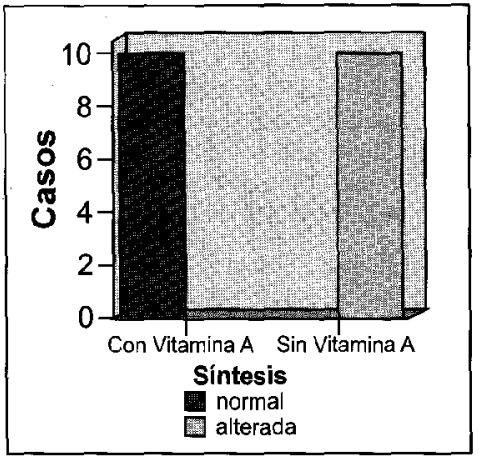

Es signifcante la alteración d la remodelación ósea en la cesta alveolar por carencia de Vitamina A

* $x^{2}$ obs $=7.273 p=0.007$ Fisher $=0.0002$ $\mathrm{p}<0,01$

* Con factor de correción.

Gráfico 5. Cambios morfológicos en el hueso alveolar

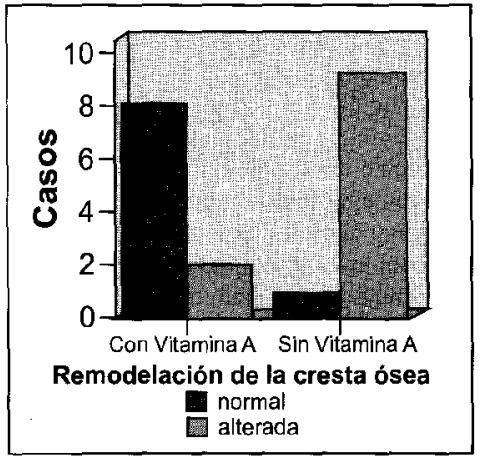

Es significante la alteración de la remodelación osea en la cresta alveolar por carencia de vitamina $A$.

* $x^{2}$ obs $=7.273 p=0.007$ Fisher $=0.0002$ $p>0.01$

* Con factor de correción.

Gráfico 6. Cambios morfológicos en el cemento radicular

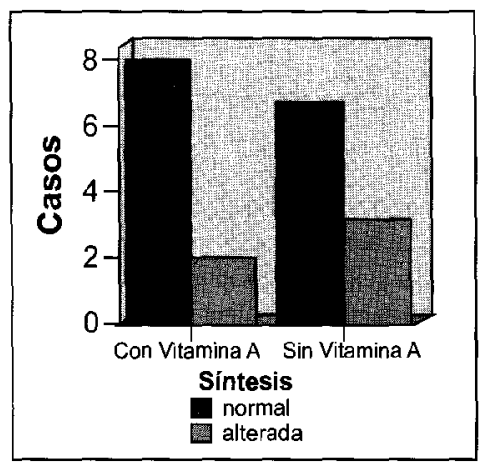

No es significantes la inhibición de la síntesis de cemento en estados carenciales de Vitamina $A$

* $x^{2}$ obs $=0 p=1$ Fisher $=0.05 p>0.01$

* Con factor de correción.
Gráfico 7. Cambios morfológicos en el ligamento peiodontal

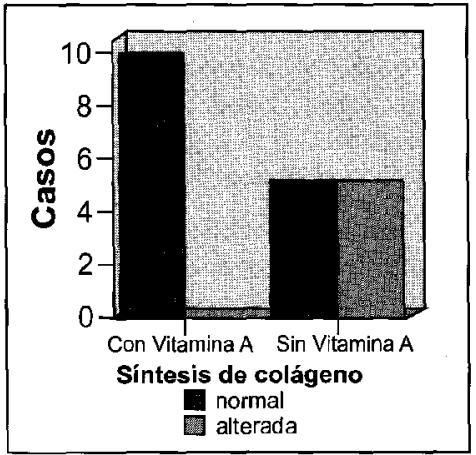

Es signifcante la inhibición de síntesis de colágena en estados carenciales de Vitamina A a nivel de confianza $95 \%$

* $x^{2}$ obs $=4.267 p=0.01$ Fisher $=0.04 p<0.05$ * Con factor de correción.

\section{Discusión}

Estudios anteriores ${ }^{5,6}$, demostraron que la deficiencia de vitamina $A$ influye alterando la diferenciación celular durante el proceso del desarrollo embrionario. No hay información hasta el momento acerca de estos procesos en estadios postnatales, producidos a nivel del tejido dentario. En éste estudio, que reúne estas características, luego de 7 a 8 semanas de avitaminosis $A$ experimental, el proceso de diferenciación celular no tiene carácter regresivo; es decir, las células no retornan a la condición de indiferenciados, salvo la alteración morfológica que conlleva en la mayoría de los casos la pérdida de sus conexiones plasmáticas laterales distales y la disminución de la altura celular, que indican una sustancial disminución de la actividad metabólica (Gráficos 1,2; Fig.1). Además, nuestros resultados muestran una mayor resistencia de los odontoblastos bucales a la carencia de vitamina $\mathrm{A}$, la cual influye considerablemente en la diferenciación de odontoblastos linguales (Gráfico 3; Fig.1). Esta situación, posiblemente se deba al factor, tiempo de diferenciación, que obviamente son diferentes para ambos odontoblastos; siendo los bucales, los que se diferenciaron antes que los linguales.

No se observó atrofia del órgano del esmalte ni cambios metaplásicos en los ameloblastos, reportados por otras investigaciones ${ }^{10-14}$. Probablemente por el momento postnatal en el que fue sometida la muestra, a dieta carente de vitamina A. Obviamente cuando el órgano del esmalte ya fue formado a los 17 días in útero, aproximadamente.
Los ameloblastos y odontoblastos alejados de la base odontogénica no sufrieron cambios aparentes, en nuestras observaciones, lo que demuestra que la falta de vitamina $A$ influye seriamente en las etapas iniciales y en el mismo proceso de diferenciación y no después; sin embargo, algunos casos mostraron un epitelio ameloblástico con cierre prematuro de compartimentos intercelulares y proximales, aparentemente asociados a la falta de vitamina A, que quizás influyan en los mecanismos histofisiológicos de maduración y calcificación del esmalte.

La producción de matriz adamantina ácido resistente se mostró sustancialmente inhibida en la base odontogénica, aumentando aproximadamente hasta $3 \mu$ de grosor en las partes alejadas (Fig.1). Deulofeu y Marenzi ${ }^{17}$ mencionaron similares resultadosA en la deficiencia primaria severa de vitamina A en perros, incluso con atrofia de ameloblastos y por consiguiente, con ausencia de esmalte. Los estudios de Boyle ${ }^{15}$ y Dinnerman ${ }^{15}$, en gérmenes dentarios de niños con hipovitaminosis $\mathrm{A}$, han demostrado cambios del retículo estrellado a una capa de células escamosas, supuestamente con graves alteraciones del ameloblasto $y$ de la producción del esmalte.

La información cruzada de estudios anteriore $s^{15-17}$ y la nuestra, indican que la falta de vitamina $A$, no solo influye en el desarrollo prenatal del esmalte, si no que además sugiere que el estado carencial después de la diferenciación celular, influye en los niveles cuantitativos de la producción del esmalte (Fig.1). La escasa resolución del microscopio óptico no permite la evaluación cualitativa

El engrosamiento dentinario por labial y disminución de la misma por lingual con desorientación de odontoblastos y formación de dentina defectuosa en ratas con hipovitaminosis $A$, observado por primera vez por Wolbach y Howe $e^{8,9}$, ampliamente confirmado por numerosas investigaciones ${ }^{1074}$, fue algo confuso en los sitios alejados de la base odontogénica, y muy claro en el lado lingual de la base, con ausencia de odontoblastos (Fig.1). La causa de este último por atrofia o por falta de diferenciación, es desconocido; sin embargo, podría atribuírsele a la falta de acciones moleculares de la vitamina A en los núcleos de los odontoblastos que activan a los receptores alfa y gama del ácido retinoico, que a su vez activan 
genes homeobox, en etapas iniciales del desarrollo dentario. Además, se observó una significante disminución del grosor de la pared dentinaria bucal en la zona germinativa (Fig.1).

En la mayoría de los casos, luego de 7 a 8 semanas de estado carencial do vitamina $\mathrm{A}$, el complejo periodontal presentó un engrosamiento inusual de la cresta alveolar sin resorción ósea (Fig.3), coincidiendo de alguna forma con los resultados de Irving ${ }^{11,12}$; no obstanle que ningún caso manifestó una desordenada hiperproducción osteoblástica.

La reacción conectiva del periodonto, que Marenz $\mathrm{i}^{17}$ menciona, no fue categórica (Gráfico 7). Más bien, en algunos casos el conectivo fue a delgados haces de fibras colágenas.

La actividad proliferativa post natal de fibroblastos en el ligamento periodontal, está ligeramente aumentada en la avitaminosis $\mathrm{A}$; siendo más notable en elapas tempranas del desarrollo. ${ }^{6}$

La prueba de significancia estadística de los cambios morfológicos del complejo dentoalveolar en avitaminosis $\mathrm{A}$ a un nivel de confianza del $99 \%$, resultó positiva; a excepción del cemento radicular que fue No Significativa y. del ligamento periodontal, que tuvo carácter presuntivo, a pesar del engrosamiento del cemento y la reacción fibroblástica del ligamonto, presentes en ambos grupos experimentales; causada probablemente por otros factores no determinados en éste estudio

\section{Conclusiones}

La vitamina A es esencial en los mecanismos histofisiológicos de las células diferenciadas, presentes en el desarrollo post natal del tejido dentario. Es por ello que los especimenes alimentados con dieta carente de vitamina $\mathrm{A}$ muestran trastornos dentoalveolares, tales como:

a) Cambios morfológicos de los ameloblastos consistentes en pérdida de conexiones plasmáticas laterales. distales y pérdida de la altura celular del ameloblasto.

b) Se inhibe la producción de esmalte post natal cn estados carenciales de vitamina $A$. Las pruebas estadísticas son altamente significativas.

c) Cambios morfológicos de los odontoblastos: alteración en la formación de organelas productoras de proteínas. Disminuye la producción dentinaria en el lado bucal de la base odontogénica por avitaminosis A. las pruebas estadísticas son significativas.

No existe diferenciación de odontoblastos linguales, ni producción de dentina correspondiente en la base odontogénica por carencia de vitamina $\mathrm{A}$. Las pruebas estadísticas son altamente significativas.

d) Cambios morfológicos en la matriz dentinaria: disminuye la producción dentinaria por inhibición de sintesis proteica en el lado bucal de la base odontogénica por avitaminosis A. Las pruebas estadísticas son altamente significativas.

e) Cambios morfológicos en el hueso alveolar presentando engrosamiento óseo de la cresta alveolar con alteración de la remodelación.

f) Reacción fibroblástica del ligamento periodontal.

\section{Referencias}

1. Arroyane, G.: Interrelations between protein and vitamin $A$ and metabolism. Am.J.Clin.Nutr. 1969, 22:1119.

2. Olson, J.A.: Metabolism and function of vitamin A.Fed.Proc. 1969, 28:1670.

3. Roels, O.A.: Vitamin A phisiology. 1970, JAMA, 214:1097.

4. Dennert, G.: Retinoids and the immune system: Immunostimulation by vitamin A in: The Retinoids, Academic Press, Orlando, FL. 1984, vol. 2 (M.B. Sporn, A.B. Roberts,and D.S. Goodman,eds.), pp 373-390
5. OPS-ILSI: Conocimientos actuales sobre nutrición. Publicación Científica No532,sexta Edic,1991. págs 113-135.

6. Azano, M.A.;Lamb, A.J.;\& Olson,J.A.: Growth, appetite, sequence of pathological signs and survival following the induction of rapid, synchronous vitamin A deficiency in the rat. J.Nutr. 1979, 109:1419-1431.

7. West, et al.: Vitamin A deficiency, infection and immune function: studies using the chicken as model. Summary XIV, IVACG meeting, Quito, 1992. pág 105.

8. Wolbach, S.B. and Howe, P.R.: Tissue changes following deprivation of fat soluble A vitamin. J.Exp.Med. 1925, 42:753-778.

9. _-. The incisor teeth of albino rats and guinea-pigs in vitamin A deficiency and repair. Amer. J. Path. 1933, 9: 275-292.

10. Cagnone Leroy D.: Trastornos vitamínicos-Patología Oral de Thoma,Gorlin y Goldman. EditSalvat,Barcelona. Edic 1984, págs 667-676.

11. Irving, J.T.: The effects of avitaminosis and hypovitaminosis $A$ upon the incisor teeth and incisal alveolar bone of rats. J.Physiol. 1949, 108:92-101.

12. ‥_-: Friihe histologische veranderugen in der knochen formation bei vitamin-A-Mangel, Med. Klin. 1956, 51: 690-693.

13. Shibata, M.: Effect of the lack of vitamin on development of the teeth, JAP. J.Exp. Med. 1931, 9:21-32.

14. World Health Organization:Vitamin A deficiency and xerophthalmia (tcch. Rep. Ser. N5590) Genova: WHO,1976.

15. Boyle, P.E.: Manifestations of vitamin $\Lambda$ deficiency in a human tooth germ. J.Dent.Res. 1933,13:39-50.

16. Dinnerman, M.: Vitamin A deficiency in unerupted teeth of infants. Oral Surg.Oral Med.Oral Path., 1951, 4:10241038.

17. Deulofeu, V. y Marenzi, A.D.: Química biológica, Edit. El Ateneo, Buenos Aires, 7ma Edic. 1955. pag.567-572. 